\title{
Contextualisation et configuration discursive
}

L'image de guerre Les obus miaulent

Contextualization and Discursive Configuration. The Great War Image: Les obus miaulent

\section{Philippe Wahl}

\section{OpenEdition}

\section{Journals}

Édition électronique

URL : https://journals.openedition.org/pratiques/2505

DOI : $10.4000 /$ pratiques. 2505

ISSN : 2425-2042

\section{Éditeur}

Centre de recherche sur les médiations (CREM)

\section{Référence électronique}

Philippe Wahl, «Contextualisation et configuration discursive », Pratiques [En ligne], 165-166 | 2015,

mis en ligne le 01 octobre 2015, consulté le 08 juin 2021. URL : http://journals.openedition.org/ pratiques/2505; DOI : https://doi.org/10.4000/pratiques.2505

Ce document a été généré automatiquement le 8 juin 2021.

(c) Tous droits réservés 


\title{
Contextualisation et configuration discursive
}

\author{
L'image de guerre Les obus miaulent \\ Contextualization and Discursive Configuration. The Great War Image: Les obus \\ miaulent
}

Philippe Wahl

1 L'analyse discursive des figures ressortit à une problématique générale de contextualisation des faits de langage ${ }^{1}$. Elle justifie en retour un dépassement des répertoires rhétoriques par une définition élargie de la notion de figuralité. Tout est affaire de contexte(s), selon une dialectique de l'encodage et du décodage au cœur des orientations énonciatives et pragmatiques des disciplines du texte ${ }^{2}$. La figuralité implique des procédures de contextualisation, sous un double aspect verbal et situationnel que complexifient l'interdiscours et l'intertextualité. Elle tient à la perception de formes signifiantes sur un fond discursif, selon des grandeurs à définir en fonction de parcours interprétatifs (voir Rastier, 2001). Le contexte verbal se conçoit ainsi de manière interactive, croisant les approches: combinaison de paramètres linguistiques ; dialectique forme/mouvement impliquant la dynamique de lecture ; jeux d'échelle attentifs aux conditionnements herméneutique des faits locaux; relations entre textes régulées par les normes de genres.

2 Les enjeux de la contextualisation seront explorées à partir d'une métaphore empruntée au corpus d'images de guerre de Guillaume Apollinaire : Les obus miaulent. Après une description lexicale, la figure sera rapidement cernée dans l'interdiscours de l'argot militaire et de la littérature de guerre. Chez Apollinaire, elle apparait de manière différenciée sous divers genres (correspondance, poésie, chronique journalistique) et formes (prose/vers). On insistera sur le processus d'appropriation de l'image et sur son rôle dans la configuration du discours poétique. La création verbale exploite l'expérience du front à travers la médiation de codes culturels, au service d'une esthétique singulière. La problématique se double d'un questionnement éthique, les poèmes de guerre d'Apollinaire ayant soulevé la controverse, en particulier parmi 
les surréalistes. À travers la posture complexe d'un poète soldat et amoureux, l'écriture implique une éthologie de la figure dans la représentation de la guerre (voir Wahl, 2010).

\section{I. «Les obus miaulent » : devenir-cliché d'une métaphore}

3 L'étude des conditions de mise en discours de l'image suppose un détour par le potentiel sémantique du verbe et de ses dérivés. Dans le domaine militaire, la métaphore tend vers le cliché, sous le double aspect d'image devenue banale et de collocation verbale. Un enjeu du discours littéraire est d'exploiter ce matériau, en le remotivant ou en le renouvelant.

\section{Lexique et rhétorique : figure et sens figuré}

4 La métaphore verbale se distingue par le caractère suggestif du transport figural. Ses analyses oscillent entre rigidité sémiotique des termes impliqués dans un sens " purement relationnel » (Tamba-Mecz, 1981:31, 184) et "remaniements sémiques » (Rastier, 2001: 164); entre "catégorisation indue» (Kleiber, 2003) et simple caractérisation analogique du prédicat (Cadiot, 2009). Ces oppositions théoriques trouvent un dépassement dans la diversité des réalisations discursives, selon le degré de lexicalisation de la figure. Le verbe miauler présente dans le Trésor de la langue française (TLF) un large répertoire de sens "figurés». Le sens premier, fondé sur l'onomatopée miaou ( (crier, en parlant du chat et de certains félins »), s'applique par analogie à d'autres animaux, aux personnes - souvent péjorativement, d'où la spécialisation de sens " gémir, se lamenter "-, mais aussi aux choses $^{3}$, en particulier un instrument de musique, une arme ou un projectile :

«Les balles miaulent au-dessus de la tranchée, très bas, et plusieurs claquent sur le parapet, comme des coups de fouet » (Dorgelès, $1964: 100$ ).

L'obus n'est pas mentionné, mais il apparait sous forme dérivée dans l'argot militaire :

«Miaulant, -ante, part. prés. et adj. Qui miaule. [...] En partic., arg. milit. Sont miaulants tantôt le gros obus tantôt la torpille aérienne, jamais un canon (Esn. 1966). Subst., p. ell. du déterminé. "Miaulant, obus allemand de 77 fusant" (Dauzat, $2007: 271)$.

Miaule, subst. masc. a) Arg. milit. Synon. de miaulant» ([...] v. Esn. Poilu 1919, p. 353).

6 Le TLF illustre l'emploi militaire du nom déverbal suffixé chez Cendrars :

« Derrière nous c'était la pagaïe des régiments décimés, des isolés qui se trottaient [...], des grenades qui éclataient, des explosions [...] et les miaulements fous des 75 qui s'acharnaient à vouloir raser et nettoyer la crête que nous avions conquise " (Cendrars, $1946: 86$ ).

\section{Argot de la guerre}

7 Les spécialisations du verbe miauler dans les discours du front assignent à l'actant un paradigme restreint: la balle ou l'obus, voire le canon par métonymie. Cette stabilité combinatoire favorise le figement de la figure en clichét. Or la problématique du cliché est en affinité avec celle de l'argot : visée expressive du discours (menacée par l'usure) ou spécificité sociolectale? 
8 Dans son enquête sur «l'argot de la guerre», A. Dauzat (2007: 55) corrige la représentation d'" une langue créée de toutes pièces ", liée au stéréotype du " poilu ». De fait, ce langage est nourri de l'argot des casernes mais aussi de l'argot parisien, et varie selon l'origine des soldats, selon les secteurs militaires. Mais le mythe séduit dès le début du conflit une presse avide de couleur locale, qui contribue au «bourrage de crâne ». La Grande Guerre a jeté dans la mêlée des intellectuels qui font de l'argot du front un vecteur d'expression, mais aussi un objet d'étude. Sa pratique se double ainsi d'une conscience linguistique et littéraire ${ }^{5}$.

\section{a) Composante expressive}

9 L'emploi figural du verbe miauler repose sur un schème psycholinguistique d'animation de l'inanimé. Le potentiel expressif du trait /animal/ est susceptible d'être renforcé par un trait de sauvagerie. Son étrangeté implique le lecteur dans une expérience partagée. C'est un aspect souligné par P.Cadiot, discutant l'analyse par G. Kleiber (2003) de l'énoncé Le chien miaule :

"Le transfert analogique d'emploi ne traduit pas tant une catégorisation indue qu'un glissement de la prédication vers la sphère de l'expérience sensible du sujet entendant (un son plutôt strident, insistant, trop aigu, légèrement angoissant, sans doute parce que facilement ressenti comme plaintif). La métaphoricité est inséparable d'une recharge du sens du verbe dans le champ phénoménal du sujet entendant, voire même d'un ressourcement aux bases auditives de l'onomatopée dans ce cas particulier $»^{6}$.

10 Le potentiel figural du verbe est décuplé par l'intensité et l'ampleur du phénomène sonore de l'explosion d'obus. De fait, les discours du front mettent en scène un bestiaire plus ou moins fantastique, fondé sur des impressions et des associations mentales codifiées ${ }^{7}$.

\section{b) Limites de l'expressivité : usage et usure}

11 L'expressivité d'une métaphore est réduite par la progressive lexicalisation de « sens figurés ». Mais en l'occurrence la motivation sémantique du verbe reste forte. Significativement, le TLF propose peu de gloses lexicographiques, renvoyant implicitement au sens typique ("crier, en parlant du chat et de certains félins»). La glose rapportée à l'humain, "gémir, se lamenter », infléchit l'image dans le registre pathétique (cf. «De temps en temps un obus se lamente», LL 125 : 293). Par ailleurs, la fréquence de la métaphore l'affaiblit comme schème dynamique et conforte son statut de cliché. Mais cette appréhension déplace la problématique, interrogeant la valeur du cliché en tant que tel, comme indice d'un type de discours ou de registre (voir Riffaterre, 1971 : 161 sq.). Et la figure se prête à des remotivations en contexte, ici avec un saisissant effet de variation lexicale (miauler/jurer comme un chat)

« Notre artillerie répondait - soixante-quinze miaulant, cent vingt brutal et le canon-revolver, qui jure comme un chat » (Dorgelès, 1964 : 224).

\section{c) Spécification lexicale}

12 Une autre cause d'affaiblissement de l'image est la fonction pragmatique de discrimination des projectiles, bien attestée dans les discours du front ${ }^{8}$. Le miaulement se charge alors d'une valeur spécifiante : 
«Les oreilles s'habituent vite à ce roulant fracas. On les reconnaît tous, rien qu'à leur voix : le soixante-quinze qui claque rageur, file en miaulant et passe si vite qu'on le voit éclater quand on entend le départ; le cent vingt essoufflé qu'on croirait trop las pour achever sa course ; le cent cinquante-cinq qui semble patiner sur des rails et les gros noirs, qui passent très haut, avec un bruit tranquille d'eau qu'on agite » (Dorgelès, $1964: 49$ ).

La corrélation entre «le soixante-quinze » et le prédicat miauler est confirmée par la dénomination le miaulant. Elle est toutefois relative : le miaulant est l'obus de 75, mais aussi l'«obus allemand de 77 fusant » (Dauzat, $2007: 250$ ). Et inversement, A. Dauzat choisit le «canon de 75 » pour illustrer la « riche synonymie » de l'argot : «À lui seul, [il] est tour à tour, - d'après son bruit l'aboyeur, le râleur, le roquet » (ibid. : 90). Sur le théâtre de la guerre, la suggestion métaphorique a raison de la taxinomie.

\section{d) Ambivalence de la valeur figurale}

L'interprétation de la figure met en jeu l'ambigüité de la notion de valeur dans les disciplines du texte. L'analyse textuelle justifie l'extension de la définition différentielle du signe (in absentia) aux relations lexicales in praesentia. Par ailleurs, la figure peut être évaluée selon deux paradigmes, pragmatique et esthétique, qui fusionnent dans «la portée d'un acte sémiotique " créant "de la valeur» (Molinié, 2005: 41). En l'occurrence, l'appréciation de l'image est liée à sa composante thymique. L'analogie actualise souvent dans le cri félin des traits dysphoriques, /désagréable/, voire / inquiétant/, qui peuvent servir la composante argumentative du discours. R. Amossy a analysé chez Giono une variante de l'image portant le cliché (implicite) de la chair à canon à une cruauté paroxystique :

«Et maintenant la mitrailleuse mange quelque chose de chaud et de vivant; elle ronronne dans la chair molle » (Le Grand Troupeau, 1931, p. 115 ; Amossy, 2000 : 188).

La mutation de l'animal domestique, la réification de l'homme donnent à voir la barbarie d'une guerre mécanique, proprement inhumaine. Mais le motif félin permet, inversement, d'apprivoiser l'adversité par un effet hypocoristique. C'est aussi le rôle des onomatopées ou des surnoms, qui confèrent aux discours du front une expressivité naïve :

«D'autres obus sont dits [...], selon les variétés de sifflement, miaulant, glin-glin, zinzin (fréquent), dzin-dzin (plus rare), zim-boum » (Dauzat, $2007: 91)^{9}$.

\section{Effets romanesques}

16 Dans les récits et romans de guerre, le paradigme lexical de miauler est attesté mais peu fréquent, alors que les textes font une large place aux impressions sonores. Les occurrences apparaissent généralement dans des passages visant à l'expressivité, souvent liées à des séries verbales ou déverbales.

\section{a) Henri Barbusse, Le Feu (prix Goncourt 1916)}

17 Le chapitre "Bombardement» du Feu se distingue par une théâtralité apocalyptique, fondée sur un réseau d'images et un faisceau de caractérisations négatives :

« Dans une odeur de soufre, de poudre noire, d'étoffes brûlées, de terre calcinée, qui rôde en nappes sur la campagne, toute la ménagerie donne, déchaînée. Meuglements, rugissements, grondements farouches et étranges, miaulements de chat qui vous déchirent férocement les oreilles et vous fouillent le ventre, ou bien le long 
ululement pénétrant qu'exhale la sirène d'un bateau en détresse sur la mer. Parfois même des espèces d'exclamations se croisent dans les airs, auxquelles des changements bizarres de ton communiquent comme un accent humain" (Barbusse, 1988 : 272-273)

18 Le passage file la métaphore de la bête sauvage. Le miaulement est le quatrième d'une série de noms déverbaux, dont la scansion (homéotéleute en -ment) renforce le pouvoir suggestif. La subordonnée relative développe le trait de férocité, soutenu par les allitérations (en particulier chuintante) et les assonances (en particulier nasale) : "farouches et étranges", "déchirent férocement", "fouillent le ventre ». Cet expressionnisme valut à H. Barbusse les reproches de J. N. Cru (1993 : 564) - « une exposition de tératologie plutôt qu'un tableau des horreurs réelles que nous avons tous vues »-, qui saluait en revanche la sobriété de M. Genevoix.

\section{b) Maurice Genevoix, Sous Verdun (1916)}

19 Dans une prose mesurée combinant hypotaxe et parataxe, le verbe puis le nom sont ici employés à propos des balles. La première séquence développe l'effet initial de saturation spatiale par quatre paliers de perception.

«Partout, au-dessus de nous, devant nous, à droite, à gauche, ça siffle, miaule, ronfle, claque. À quelques pas de moi, les balles d'une mitrailleuse assourdissante arrivent dans la terre, obstinées, régulières et pressées " (Genevoix, 1996 : 37).

Plus loin, le nom vient clore une série verbale, confirmant la combinaison avec le sifflement, ainsi que l'afférence psychologique («obstinées », « coléreux ») :

«On croirait un duel étrange, innombrable et sans merci, le duel de toutes ces petites choses dures et sifflantes qui passent, passent, claquent, tapent et ricochent avec des miaulements coléreux, là, devant nous, sur la route dont les cailloux éclatent, pulvérisés » (ibid. : 104).

\section{c) Blaise Cendrars, de J'ai tué (1918) à La Main coupée (1946)}

21 L'image du miaulement révèle le travail de réécriture auquel Cendrars a soumis la prose poétique de J'ai tué, après la Seconde Guerre mondiale. Dans le texte de 1918, la métaphore du train se prolonge en un déchainement de ménagerie qui rappelle H. Barbusse, jusque dans l'écho comparatif « comme un accent humain » :

« Nous sommes sous la voûte des obus. On entend les gros pépères entrer en gare. Il y a des locomotives dans l'air, des trains invisibles, des télescopages, des tamponnements. On compte le coup double des rimailhos. L'ahanement du 240. La grosse caisse du 120 long. La toupie ronflante du 155. Le miaulement fou du 75. Une arche s'ouvre sur nos têtes. Les sons en sortent par couple, mâle et femelle. Grincements. Chuintements. Ululements. Hennissements. Cela tousse, crache, barrit, hurle, crie et se lamente. Chimères d'acier et mastodontes en rut. Bouche apocalyptique, poche ouverte, d'où plongent des mots inarticulés, énormes comme des baleines saoules. Cela s'enchaîne, forme des phrases, prend une signification, redouble d'intensité. Cela se précise. On perçoit un rythme ternaire particulier, une cadence propre, comme un accent humain " (J'ai tué, 2013, p. 15-16).

M. Frédéric a montré le contraste avec la version de La Main coupée, que distingue son ancrage "épisodique ». L'occurrence plurielle des "miaulements fous des 75 », citée par le TLF (ci-dessus), y est syntaxiquement intégrée à une séquence narrative presque pittoresque (2003: 76-77), soumise à un effet de sourdine. 
rapide aperçu sur les proses de guerre illustre la fonction pathémique d'un réseau d'images violentes ${ }^{10}$. Le motif de la « voix ", la composante psychologique des discours suggèrent un continuum entre les armes, l'animal et une humanité aliénée. Dans la scène suivante, M. Genevoix assigne le trait /sauvage/ à l'ennemi à travers le stéréotype de la langue rauque, dans l'expression d'une rage cosmique :

«"Hurrah! Vorwärts !..." / Ils s'excitent en hurlant, les sauvages. Leurs voix rauques s'entendent à travers la fusillade, déchiquetées par les détonations pressées, charriées par le vent avec les rafales de pluie. Vent furieux, pluie forcenée; il semble que la rage des combattants gagne le ciel » (Genevoix,1996 : 49).

À travers les ressorts stylistiques de la représentation, la figure interroge ainsi le lien entre esthétique et éthique, qui a joué un rôle déterminant dans la réception des poèmes de guerre d'Apollinaire.

\section{II. Écrits d'Apollinaire : appropriation d'une image}

Dès sa période de formation militaire à Nîmes, les écrits d'Apollinaire offrent des représentations de la guerre reposant sur des médiations culturelles (mythologie, histoire, littérature). Le processus d'appropriation de la métaphore Les obus miaulent coïncide avec son arrivée "en avant ", en avril 1915 (LL $116: 260$ ). Il met en jeu une stylisation conjointe du discours et de l'univers de discours, conforme au principe énoncé par Jakobson :

«Toute expression verbale stylise et transforme, en un certain sens, l'événement qu'elle décrit. L'orientation est donnée par la tendance, le pathos, le destinataire, la

"censure" préalable, la réserve des stéréotypes " (Jakobson, 1977 : 41).

\section{Situation et stylisation}

Le statut paradigmatique des figures de rhétorique manifeste plus sensiblement le rapport entre perception et conception dans l'émergence de formes signifiantes en contexte. Cette composante phénoménologique peut être traitée en termes de Gestalt dans le continuum du discours (Rastier, $2001: 165$; Bonhomme, $2000: 148$ ). Elle trouve une justification particulière dans le schème qu'implique l'obus: tir > trajectoire > explosion > effets destructeurs. Son potentiel synesthésique (vision, ouïe, odorat, tact) est propice à la création d'esthésies en affinité avec la visée du discours (Rastier, 2001 : 163).

Sous la plume d'Apollinaire, la stylisation procède d'une rigoureuse sélection parmi le champ lexical des bruits de guerre. La collocation d'un actant et d'un procès typiques (Les obus miaulent) conforte l'effet de cliché. Il est toutefois compensé par le caractère circonscrit des occurrences, qui contraste avec les combinaisons et accumulations de la prose narrative. L'image manifeste son rôle dans la configuration du discours et de l'univers de discours, qui fait interagir divers modes de contextualisation.

a. contexte situationnel parfois mentionné en titre ( "Venu de Dieuze », "La nuit d'avril $1915 »)$, dont les contours sont soumis à un sensible « éclairage » poétique ${ }^{11}$. En avril 1915, le canonnier Kostrowitzky est affecté à un échelon d'artillerie, puis nommé « agent de liaison » sans être exposé en première ligne. Cette distance relative favorise une appréhension déréalisante, voire esthétisante du spectacle de l'artillerie ${ }^{12}$;

b. contexte énonciatif caractérisé par l'ethos complexe de l'épistolier. Ses deux destinataires principales, Lou puis Madeleine, lui font parcourir les gammes du discours amoureux. 
Apollinaire fait aussi valoir un statut de poète-soldat, liant l'expérience du front à l'écriture, comme pratique immédiate ou projet littéraire à nourrir d'«impressions toutes fraîches, prises sur le vif » (LL $121: 279)$;

c. contexte interdiscursif manifesté dès la période de formation militaire à Nîmes, où l'imaginaire se nourrit de topiques classiques et de l'interdiscours militaire (« Mais ce pâle blessé m'a dit à la cantine / Des obus dans la nuit la splendeur argentine », Po: 211). L'expérience $\mathrm{du}$ front contribue à amplifier des thèmes anciens de la poétique apollinarienne, en particulier dans sa veine érotique et cosmique ;

d. contexte historique de la Grande Guerre, sollicitant un lexique technique. L'érudition d'Apollinaire trouve une nouvelle ressource dans l'argot du front, qui s'affiche dans les titres de Calligrammes : titre de section ("Case d'armons " ${ }^{13}$ ) ou de poème ("Saillant », "Échelon », « S P »). La compétence langagière était variable entre le front et l'arrière. À cet écart initial s'est ajoutée la distance temporelle, qui renforce certaines opacités ponctuelles. Au-delà d'effets de couleur historique, celles-ci mettent en jeu la composante philologique de la lecture.

É. Benveniste (1974 : 79 sq.) a défini l'énonciation comme "un procès d'appropriation » de la langue par une "réalisation individuelle». Cette description présente une motivation spécifique dans le domaine stylistique, comme espace de singularisation du discours (voir Jaubert, 2007). Le poète est toutefois susceptible d'inverser la perspective, à la manière de P. Reverdy (1948 : 152) soulignant la fonction médiatrice du langage: "C'est grâce aux mots, grâce au langage, grâce aux images que l'homme s'approprie le monde extérieur ». L'énonciation littéraire fonde surtout un ordre de discours autre, approprié à ses visées.

\section{Lettres à Lou}

Les sentiments d'Apollinaire pour Lou expliquent une confusion des univers, que développe l'expérience de la guerre. Sur fond d'artillerie, l'image du miaulement subit une inflexion amoureuse exploitant l'ambivalence thymique inhérente à la passion. Ses modes de contextualisation apportent un éclairage sur l'écriture du poète-soldat, variable selon les genres et les formes.

\section{a) Variations amoureuses}

30 La première occurrence du motif apparait le 8 avril, au lendemain de l'arrivée sur le front. Elle est précédée de ce tableau portant la marque de la convention poétique :

«C'est une vie grandiose qui ne va pas sans une mélancolie lyrique extraordinaire.

Les obus gémissent d'une façon déchirante » (LL 117 : 263).

31 À la page suivante, le gémissement fait place au miaulement, dans une prédication par paliers :

«Mon Lou, la voix des obus est un véritable miaulement, ils miaulent comme des

chats amoureux. C'est fantastique » (ibid. : 264).

32 Le groupe sujet de la première proposition présuppose un trait/vocal/ auquel l'attribut nominal apporte une spécification animale renforcée par l'enclosure véritable (absente en poésie). La seconde est relancée par la variante verbale de la métaphore, qu'authentifie une comparaison explicitant l'afférence /féline/ par la suggestion du rut. L'intensité du phénomène est consacrée par la clausule superlative: "C'est fantastique ». La composante explicative et évaluative de la prose épistolaire s'allie à 
des signes de poéticité: la figure de dérivation miaul(ement) structure un chiasme encadré par deux traits à afférence humaine («voix »/« amoureux »), qui font écho à l'adresse affectueuse (« Mon Lou »). bien simple il est vrai, [d'un] véritable baptême du feu » :

" Arrivés à la hauteur de la forêt [...], un miaulement déchirant et Pan! un fusant éclate dans les arbres à 25 mètres et à 15 mètres de hauteur. Des feuilles volent. Les chevaux sont habitués. On les met au galop dans les terres sur la lisière et nouveau miaulement, Pan! à la même hauteur et un peu plus près mais pas dans les arbres, si bien qu'on a vu la fumée jaune-rougeâtre qui est restée longtemps en l'air » (LL $121: 279)$.

38 (parataxe, énoncés nominaux, onomatopées). Le nom déverbal, fréquent dans les romans de guerre, sert une phénoménologie tendant à effacer l'actant (occurrence disjointe d'«un fusant»). Au-delà d'une épithète conventionnelle ("déchirant»), l'expression de la violence ("éclate», "volent») est circonscrite à l'unité d'un événement, voire désamorcée ("Les chevaux sont habitués»). La composition du passage stabilise le schème discursif : miaulement > Pan!, jusqu'au suspens visuel final. 
L'expressivité ne vise pas ici l'amplification épique, mais donne une vision presque inoffensive de l'aventure.

\section{Vers libre} mètre en conservant parallélismes et répétitions. « Agent de liaison » offre une version intériorisée de la canonnade, portée à l'hyperbole à la faveur d'une syllepse sur ardente :

« Mon cœur est comme l'horizon où tonne et se prolonge

La canonnade ardente de cent mille passions

Ah ! miaulez. Ah ! miaulez les chats d'enfer » (LL $123: 284)$.

Le trait infernal de l'adresse trouverait une clé dans l'«air étrangement diabolique » d'une amante infidèle (LL $118:$ 266). Une variante apparait dans l'exhortation de la 《 $43^{\mathrm{e}}$ batterie du $38^{\mathrm{e}}$ » du poème choral « Scène nocturne du 22 avril 1915 »:

"Les chevaux hennissent Éteignez les lumières

Les caissons sont chargés Empêchez les hommes de dormir

Entends miauler les tigres volants de la guerre » (LL $132: 312$ ).

41 Les deux métaphores nominales ("chats d'enfer", "tigres volants»), variations paradigmatiques sur le syntagme "chats volants", produisent un effet déréalisant. Mais le déchainement féroce n'est ici qu'une fantasmagorie nocturne animée par la frustration du sentiment et du désir.

Dans la correspondance, l'image se fait insistante. Elle offre une stylisation des phénomènes du front, soumis à une visée érotique qui transcende les sentiments pour dire une vérité de la guerre. Puissance, cadence, violence des tirs : les prestiges de la guerre mécanique exaltent l'imaginaire, déployant les ressorts métonymiques de la métaphore: d'un texte à l'autre, l'obus se fait lettre, amour, cœur, baiser. Il est un vecteur lyrique dans «le tonnerre des artilleries qui accomplissent le terrible amour des peuples $»^{14}$. L'ambivalence thymique (séduction ou effroi ?) relie l'animal, les armes et la femme, dans un domaine vertigineux où les rôles se confondent : «Je voudrais que tu sois un obus boche pour me tuer d'un soudain amour » (LL $132: 310)^{15}$.

\section{Configuration poétique}

43 Le motif de l'obus joue un rôle crucial dans l'élaboration poétique de "La nuit d'avril $1915 »$ (Po : 243-244). Ce texte, dont on connait plusieurs variantes, clôt la plaquette Case d'armons en "une synthèse remarquable des procédés poétiques " (Debon, 2008: 222 sq.). L'évolution de la mise en espace des quintils d'alexandrins, par segmentation et détachement, contribue à la dramatisation de la scène jusqu'à la fragmentation finale. On retiendra pour ce rapide examen la version publiée dans Calligrammes, qui mériterait une comparaison avec celle initialement adressée à Lou (LL 120 : 273-274) ${ }^{16}$.

Le texte a été salué par A. Breton (1988) ${ }^{17}$ comme un bel exemple de "transmutation poétique », qui «s'empreint au firmament de la guerre». L'image du miaulement y contribue, par son indexation sur une isotopie musicale et chorale en affinité avec le lyrisme du discours. La tendance au vers-énoncé fait de la prédication métaphorique un micro-acte de langage au service de la sémiosis textuelle, dans une dialectique de la discontinuité et de la continuité. Son lien avec l'ethos poétique peut être saisi à différents paliers. 


\section{a) Palier textuel} métamorphose. Le premier vers, "Le ciel est étoilé par les obus des Boches ${ }^{18}$, est soumis à une réinterprétation militaire par la saillance à la rime d'un référent détonnant. Mais le spectacle se prolonge en phénomène musical par le biais d'un autre cliché : le bal de l'artillerie. Le dispositif imageant des deux quintils suivants a été très retravaillé. Le deuxième condense obus, astre et cœur en une représentation ambivalente de l'éclatement.

«Comme un astre éperdu qui cherche ses saisons

Cœur obus éclaté tu sifflais ta romance.»

La confusion référentielle est redoublée par une tension aspectuotemporelle (participe accompli vs imparfait sécant). Le verbe siffler est un prédicat typique de l'obus, mais aussi une réminiscence du voyou sifflotant de "La Chanson du mal aimé », autre romance. Le motif de l'obus trouvera une variation métonymique dans les « orgues » de la clausule, associés aux « fétus de la paille » qui offrent au poète-soldat une échappée onirique entre microcosme et macrocosme.

\section{b) Palier mésotextuel : le troisième quintil}

47 Le pouvoir configurant de l'image se manifeste par sa double occurrence au cœur du poème, qui structure le quintil. La diérèse conforte son assise poétique dans l'hémistiche, surdéterminée par la disjonction du quatrième vers.

« Les obus miaulaient un amour à mourir

Un amour qui se meurt est plus doux que les autres

Ton souffle nage au fleuve où le sang va tarir

Les obus miaulaient

[Entends chanter les nôtres

Pourpre amour salué par ceux qui vont périr ».

L'insistance paradigmatique de l'image renforce un conflit temporel entre l'imparfait, hérité de la prédication précédente («sifflais »), et un contexte au présent, entre valeur gnomique et actualité à visée prospective. Cette instabilité, renforcée par celles de la personne et du nombre (ils vs tu), prépare aussi une lecture dissimilatrice (voir cidessous).

\section{c) Palier microtextuel : « Les obus miaulaient un amour à mourir »}

L'alexandrin confère les attributs de la poésie au cliché, qu'il remotive par enjambement interne suivant divers paramètres.

- syntaxique: le verbe présente une transitivité exceptionnelle, qui déplace l'accent sur le complément, le cri animal s'élevant à un mode d'expression lyrique (voir «tu sifflais ta romance $")^{19}$;

- rythmique : la structuration anapestique, surdéterminée par la diérèse sur « miaulaient $»^{20}$ et la récurrence de $/ \mathrm{m} /$ dans trois mesures, soutient la cohésion discursive de l'énoncé ;

- phonique : l'allitération en $/ \mathrm{m} /$ recouvre une transition consonantique de liquides $(/ \mathrm{l} />$ / $\mathrm{r} /)$; une transition vocalique $(/ \varepsilon / / \mathrm{o} />/ \mathrm{a} / / \mathrm{u} /)$ prépare la paronomase amour/mourir, qui fait ici écho à « miaulaient».

Pratiques, 165-166 | 2015 
- sémantique: la caractérisation infinitive à mourir remotive la métaphore féline par l'expression hyperbolique du rut. Ce tour apollinarien relie la topique amoureuse aux réalités guerrières ${ }^{21}$. Son ambivalence sémantique et thymique est corrélée à une neutralisation de la diathèse verbale inhérente à l'infinitif. Or le vers suivant engage l'amour lui-même dans le procès, actualisé sous l'aspect progressif d'un présent gnomique :

«Un amour qui se meurt est plus doux que les autres ». périphrase («où le sang va tarir»; " par ceux qui vont périr ») - et par extension aux amants $^{22}$. Le discours se nourrit de réminiscences: intertextualité interne (nageurs morts de la "Chanson du mal aimé », voix obsédante de "Nuit rhénane ») et externe, héroïque («morituri te salutant ») ou évangélique (« Mon âme est triste à en mourir », Matthieu : 26, 38).

51 L'interrelation de la guerre et de l'amour est portée au lyrisme par le lexique de la musique et du chant. Mais l'ambivalence de cette visée est confirmée par la réinterprétation du cliché, à la faveur d'une disjonction métrique qui est aussi énonciative et sémantique.

« Les obus miaulaient

[Entends chanter les nôtres ".

Le micro-système verbal (miauler/chanter) suggère une distinction entre les « obus des Boches» et "les nôtres »" Implicitement, elle réveille dans miauler le trait de sauvagerie lié au stéréotype de la langue « rauque ». L'opposition sémantique projette sur la pourpre une ironie tragique, que prolonge la dispersion finale des "orgues" dans la rêverie solitaire du soldat-poète.

53 Le discours poétique s'approprie l'image selon un double geste de désancrage référentiel et d'ordonnancement formel, qui lui confère un rôle essentiel dans la configuration du texte. Par ses déterminations propres, elle contribue à la problématisation du sens, de la référence et des valeurs, qui voue la scène poétique aux métamorphoses.

\section{Conclusion}

54 La problématique des modes de contextualisation des figures a été illustrée par une image historiquement située, selon différents genres et auteurs, mais aussi selon les variations d'une écriture particulière: celle d'Apollinaire, épistolier et poète. La métaphore fixée en cliché porte la marque d'une norme discursive, l'argot du front, qui n'exclut pas les formes de remotivation de la prose narrative, entre effet de réel et charge dramatique. Elle se prête aussi à des formes d'appropriation plus sensibles, variables selon les régimes de poéticité qu'illustre l'écriture d'Apollinaire.

La figure fonctionne comme un schème perceptuel impliquant un travail d'identification et d'interprétation, qui donne à concevoir des sens nouveaux. Ses réalisations confirment le principe herméneutique selon lequel «toute transposition d'une forme sur un autre fond modifie cette forme"; d'où "les remaniements sémiques induits par les métaphores » (Rastier, 2001 : 164). Mais ceux-ci font sens dans la mémoire des significations assignées en langue et des normes discursives. Les deux modes de signifiance, sémiotique et sémantique, distingués par É. Benveniste (1974 : 63 sq.) paraissent en interaction dans la dynamique du discours. 
La correspondance d'Apollinaire tend à estomper l'axiologie négative du verbe miauler en emploi figuré, à la faveur de l'isotopie amoureuse. Mais l'interprétation est ambivalente, comme sa valeur, conforme à sa poétique de l'« incertitude » (Po: 24). Le pouvoir de configuration de l'image fait valoir le poème comme espace emblématique de la sémiosis textuelle, caractérisé par sa densité et sa complexité sémantiques. L'énoncé métaphorique y reçoit une afférence lyrique en affinité avec le genre poétique, mais aussi avec la topique guerrière. La création instaure toutefois une distance ironique, qui affecte en retour la posture d'énonciation.

57 L'écriture invente dans le jeu des formes une mimésis de second degré : la poésie comme simulacre perceptuel. Elle trouve un ressort privilégié dans le potentiel synesthésique des impressions du front, qui imposent leur part de désordre et de déraison. Le motif de l'obus joue un rôle de catalyseur, reliant les domaines, les époques et les êtres dans une émotion répondant à l'idéal orphique d'Apollinaire. La figure, volontiers décrite comme événement local ( focal event»), implique des modes complexes de contextualisation qui peuvent engager la visée globale du texte. À cet égard, « La nuit d'avril 1915 » illustrerait les vertus d'Apollinaire « champion du poèmeévénement». La formule d'A. Breton est fondée sur la conception exigeant «de tout nouveau poème qu'il soit une refonte totale des moyens de son auteur, qu'il coure son aventure propre hors des chemins déjà tracés, au mépris des gains réalisés antérieurement " (Breton, 1952 : 23). De la figure au texte, l'appréciation d'une telle valeur implique une contextualisation large, liant auteur et lecteur dans un espace partagé de pratiques et de références culturelles.

\section{Fuvres de Guillaume Apollinaire}

APOLlinAiRe, G. (1965) : CEuvres poétiques, Paris, Gallimard, « Bibliothèque de la Pléiade » (Po).

- (1977) : CEuvres en prose complètes I, Paris, Gallimard, « Bibliothèque de la Pléiade » (Pr I).

- (1993) : Euvres en prose complètes III, Paris, Gallimard, « Bibliothèque de la Pléiade » (Pr III).

- (1990) [1969] : Lettres à Lou, Paris Gallimard, coll. « L'Imaginaire » (LL).

\section{Corpus littéraire et historique}

BARBUSSE, H., (1988) [1916] : Le Feu. Journal d'une escouade, Paris, Flammarion.

CENDRARS, B. (2013) [1946] : La Main coupée et autres récits de guerre (dont J'ai tué [1918], p. 11-18), Paris, Denoël.

COSTE (Commandant) (1929) : La Psychologie du combat, Paris, Berger-Levrault. 
CRU, J. N. (1993) [1929] : Témoins. Essai d'analyse et de critique des souvenirs de combattants édités en français de 1915 à 1928, Nancy, Presses universitaires de Nancy.

DAUZAT, A. (2007) [1918] : L'Argot de la guerre. D'après une enquête auprès des Officiers et Soldats, Paris, A. Colin.

DORGELÈs, R. (1964) [1919] : Les Croix de bois, Paris, A. Michel.

ECHENOZ, J. (2012) : 14, Paris, Éd. de Minuit.

GENEVoIX, M. (1996) [1916] : Sous Verdun, août-octobre 1914, Paris, Flammarion.

JÜNGER, E. (2008) [1920] : Orages d'acier, Journaux de guerre I, 1914-1918, trad. du français par H. Plard, Paris, Gallimard.

\section{Références théoriques et critiques}

AMossy, R. (2000) : L'Argumentation dans le discours. Discours politique, littérature d'idées, fiction, Paris, Nathan.

AMOSSY, R. \& HERSCHBERG PIERROT, A. (1997) : Stéréotypes et clichés. Langue, discours, société, Paris, Nathan.

ARAGON, L. (1964) [1935] : « Beautés de la guerre et leurs reflets dans la littérature », Europe, 421-422, mai-juin, p. 132-137.

BENVENISTE, É. (1974) : Problèmes de linguistique générale II, Paris, Gallimard.

BONномmE, M. (2005) : Pragmatique des figures du discours, Paris, H. Champion.

BRETON, A. (1969) [1952] : Entretiens (1913-1952), Paris, Gallimard.

- (1988) [1917] : « Guillaume Apollinaire », Les Pas perdus, CEuvres complètes I, Paris, Gallimard, p. 203-207.

CADIOT, P. (2009) : « Jugement thétique et métaphore prédicative », Faits de langues, 31-32, p. 109-119.

DEBON, C. (2008) : Calligrammes dans tous ses états. Édition critique du recueil de Guillaume Apollinaire, Clamart, Éd. Calliopées.

DÉTRIE, C. (2001) : Du sens dans le processus métaphorique, Paris, H. Champion.

DURANTI, A. \& GOODWIN, C. (dirs) (1992) : « Rethinking context: an introduction », in: Rethinking context. Language as an Interactive Phenomenon, Cambridge, University Press, p. 1-42.

FRÉDÉRIC, M. (2003) : « Hybridation et transposition chez Blaise Cendrars : de J'ai tué à La Main coupée ", Protée, vol. 31, 1, p. 71-80.

GOURMOND, R. de (1899) : Esthétique de la langue française. La déformation. La métaphore. Le cliché. Le vers libre. Le vers populaire, Paris, Mercure de France.

JAKOBSON, R. (1977) : Huit questions de poétique, Paris, Éd. Le Seuil.

JAUBERT, A. (2007) : « La diagonale du style. Étapes d'une appropriation de la langue », Pratiques, 135-136, p. 47-62.

KLEIBER, G., (2003) : « Sémantique lexicale : traits catégoriels ou traits non catégoriels ? », in : J.L. Aroui (dir.), Le sens et la mesure. De la pragmatique à la métrique. Hommages à Benoît de Cornulier, Paris, H. Champion, p. 99-122. 
MOLINIÉ, G. (2005) : Hermès mutilé. Vers une herméneutique matérielle. Essai de philosophie du langage, Paris, H. Champion.

PURNELLE, G. (2006) : « Les motifs concrets du front dans l'expression lyrique d'Apollinaire », in : C. Debon (dir.), L'Écriture en guerre de Guillaume Apollinaire, Clamart, Éd. Calliopées, p. 95-106.

RICHTER, M. (2006) : « La nuit d'avril 1915 », in : C. Debon (dir.), L'Écriture en guerre de Guillaume Apollinaire, Clamart, Éd. Calliopées, p. 121-148.

RABATEL, A. (dir.) (2008) : Langue française, 160, « Figures et point de vue ».

RASTIER, F. (1998) : « Le problème épistémologique du contexte et le statut de l'interprétation dans les sciences du langage ", Langages, 129, p. 97-111.

- (2001) : Arts et sciences du texte, Paris, Presses universitaires de France.

REVERDY, P. (1948) : Le Livre de mon bord. Notes 1930-1936, Paris, Mercure de France.

RICœUR, P. (1975) : La Métaphore vive, Paris, Éd. Le Seuil.

RIFFATERRE, M. (1971) : « Fonction du cliché dans la prose littéraire », in : Essais de stylistique structurale, Paris, Flammarion, p. 161-181.

TAMBA-MECZ, I. (1981) : Le Sens figuré. Vers une théorie de l'énonciation figurative, Paris, Presses universitaires de France.

WAHL, P. (2006) : « Les liens du sens dans la poésie d'Apollinaire. Trois états de la syllepse », in : Y. Chevalier \& P. Wahl (dirs), La Syllepse, figure stylistique, Lyon, Presses universitaires de Lyon, p. 299-320.

- (2010) : «Éthologie de la figure : style et postures », in : L. Bougault \& J. Wulf (dirs),

Stylistiques?, Rennes, Presses universitaires de Rennes, p. 209-228.

\section{NOTES}

1. Pour une mise en perspective épistémologique de la notion de contexte, voir F. Rastier (1998). Son point de vue herméneutique inverse les traditions d'analyse fondées sur le signe, pour faire valoir le rôle instituant du contexte: "les unités sont identifiées (pour l'expression) et sémantisées (pour le contenu) au cours de parcours interprétatifs qui se déploient sur le texte » (ibid. : 101). Dans la diversité de ses approches, la pragmatique a contribué à une définition relative et dynamique $\mathrm{du}$ contexte, attentive aux interactions entre "focal event» et "background ", mais privilégiant souvent l'actualité de la situation d'énonciation (voir A. Duranti \& C. Goodwin, 1992).

2. Voir en particulier M. Bonhomme (2005) et A. Rabatel (2008). Sur la métaphore, voir C. Détrie (2001).

3. Cet exemple d'emploi inanimé produit une forte motivation contextuelle de l'afférence dysphorique du verbe ( affreux »), par gradation du cri animal : « Malgré le grincement affreux des roues, qui sanglotaient, miaulaient, rauquaient, râlaient, tout le monde s'endormit» (Gautier, Fracasse, 1863, p. 77).

4. On peut rappeler la distinction proposée par R. de Gourmont (1899: 280), à l'appui de sa critique du cliché littéraire : «Cliché représente la matérialité même de la phrase ; lieu commun, plutôt la banalité de l'idée » (voir Amossy \& Herschberg Pierrot, 1997 : 9 sq.).

5. Voir cette mise en abyme sous la plume d'Apollinaire : «Tandis que le poète écoutait les obus miauler au-dessus des hypogées que creusent les soldats, une Dame merveilleuse caressait son 
collier d'hommes attentifs " (Le Poète assassiné, Pr I: 385). Pour les références aux œuvres d'Apollinaire, voir la bibliographie finale.

6. Cette composante phénoménale justifie selon P. Cadiot (2009) une lecture thétique: "C'est l'énoncé global qui évoque quelque chose qui m'arrive à moi, sujet percevant. »

7. Dans In Stahlgewittern (Orages d'acier), E. Jünger emploie parfois le paradigme lexical de fauchen «feuler », que les traducteurs rendent plutôt par celui de rugir que de miauler; ils utilisent en revanche ponctuellement celui de miauler pour heulen « hurler, pleurer ».

8. "L'obus avant d'éclater, grince ou jette dans les airs au cours de son trajet comme un long cri strident. Selon qu'il est fusant ou percutant, selon son calibre, sa vitesse, la tension de sa trajectoire, le vacarme varie depuis le bruit de la sirène jusqu'au bruit de ferraille d'un train rapide en marche. Tous les combattants avaient appris à distinguer chacun des calibres des obus, depuis les 77 allemands jusqu'au 420, par le seul ronflement, miaulement ou bruit particulier qui les caractérise » (Coste, $1929: 170)$.

9. Voir cette variante anglaise de l'onomatopée, dans un répertoire de "voix » du front où le diminutif désignant les shrapnels allemands s'inspire d'une chanson de music-hall, « Archibald, certainly not " (1911): "More disconcerting than the actual burst of the shell is the "whirr" of the splinters or, in the case of shrapnel, the loud "miaow" of the flying bullets. I suppose our gallant airmen have more shrapnel fired at them than anyone else, and the loud "clump, clump, clump" of the bursting "Archies", followed by the whine of three hundred bullets flying from each shell, is an almost continuous tune down the line even on the quietest of days » (T. J. Salmon, "The Voice of the Guns », from The Cornhill Magazine, London, in The Lotus Magazine, Vol. 8, $\mathrm{N}^{\circ} 2$ (Nov. 1916), p. 55-58; en ligne: http://www.jstor.org/stable/20543777, consulté le 9 octobre 2014).

10. Postérité paradoxale de cette poétique romanesque, réinterprétée par le narrateur de 14 de J. Echenoz (2012: 78) : «Et dès le lendemain matin ça n'a plus eu de cesse encore, dans le perpétuel tonnerre polyphonique sous le grand froid confirmé. Canon tonnant en basse continue, obus fusants et percutants de tous calibres, balles qui sifflent, claquent, soupirent ou miaulent selon leur trajectoire, mitrailleuses, grenades et lance-flammes, la menace est partout ». Mais la posture moderne se veut réflexive et critique : «Tout cela ayant été décrit mille fois, peut-être n'est-il pas la peine de s'attarder encore sur cet opéra sordide et puant. Peut-être n'est-il d'ailleurs pas bien utile non plus, ni très pertinent, de comparer la guerre à un opéra, d'autant moins quand on n'aime pas tellement l'opéra, même si comme lui c'est grandiose, emphatique, excessif, plein de longueurs pénibles, comme lui cela fait beaucoup de bruit et souvent, à la longue, c'est assez ennuyeux » (ibid. : 79).

11. "Qu'il est charmant cet éclairage / Artifice d'artificier»: le terme "éclairage » scelle l'ambivalence du poème "Roses guerrières", devenu "Fête» dans Calligrammes: effet d'« artificier » ou « artifice » poétique ? (Po : 238 ; voir Wahl, $2006: 306$ sq.).

12. D'où la critique de L. Aragon (1964) : «Fusées, signaux, grenades, c'est là cette beauté de la guerre qui fait son entrée dans la poésie, avec le cynisme de l'abstraction, où l'obus éclaté devient un cœur, mais jamais le poète ne suit la courbe de l'éclat véritable jusqu'aux vrais cœurs de chair où il va s'enfonçant. Ni sang, ni cadavres ».

13. D'abord donné en titre à une plaquette en 25 exemplaires polygraphiée «à la batterie de tir devant l'ennemi », le syntagme désigne en artillerie une pièce de rangement de la voiture caisson.

14. « Le chant d'amour » (Po : 283). Sur la polymorphie poétique de l'obus, voir G. Purnelle (2006). 15. L'équivoque du sentiment et du désir, l'ambivalence féline de la douceur et de la violence ont été exploitées par La Fontaine: "Un homme chérissait éperdument sa chatte ; / Il la trouvait mignonne et belle et délicate, / Qui miaulait d’un ton fort doux » («La Chatte métamorphosée en Femme ", Fables, Livre II, 18).

16. Le texte est donné en annexe. Pour une lecture approfondie de ce poème dédicacé à Lou, voir M. Richter (2006). 
17. A. Breton (1969: 25) reprochera ensuite à Apollinaire d'avoir éludé les "pires réalités de l'époque ", "tandis que l'esprit s'obstine on ne peut plus déraisonnablement à vouloir trouver son bien dans le "décor" de la guerre".

18. «Étoiler le ciel, lancer des fusées éclairantes » (Dauzat, 2007 : 237).

19. Une autre reprise avec changement de valence assure la continuité entre une courte pièce (antérieure) en octosyllabes ( «la cantilène du brancardier ») et le poème en vers libres «Chant de l'horizon en Champagne » dans lequel elle s'insère : «Un chien jappait l'obus miaule » > «L'obus miaule / Je te tuerai » (où la seconde proposition est interprétable comme un discours direct) (Po : 265).

20. La famille lexicale de miauler s'articule à priori en diérèse, mais l'usage est instable. Apollinaire se plait à déployer la diérèse à la rime, y compris dans un registre trivial (« Et quand l'obus miaule / On rit toujours plus fort / Tous ceux de ma piaule / Se moquent de la mort » (à Fernand Divoire, Po: 814). L'onomatopée se prête à l'allusion gaillarde, combinant dictions disyllabique et trisyllabique : «Mais nous voici à la mi-août / Ton chat dirait-il miaou / En me voyant ou bien coucou » (Po: 490).

21. "Mon petit Lou, je t'embrasse tout doucement ta langue dans ma bouche, dans un baiser à mourir » (LL $118: 268)$; « Ô mes soldats souffrants ô blessés à mourir / Cette nuit est si belle où la balle roucoule » («Chant de l'honneur », Po: 304).

22. «Les amants vont mourir » (LL $115: 257)$; «Celui qui doit mourir ce soir dans les tranchées / C'est un petit soldat mon frère et mon amant! » (« Les attentives », LL 157 : 372)

23. Dissimilation explicitée dans un poème inédit à Louis de Gonzague Frick: «Les obus miaulent en boche / Comme chats-volants en débauche / Les nôtres chantent en poilus / Intrépides et résolus » (20 avril 1915).

\section{RÉSUMÉS}

L'analyse discursive des figures relève d'une problématique générale de contextualisation des faits de langage, impliquant une définition extensive et interactive de la notion de contexte. Les procédures de mise en discours seront explorées à partir d'une image de la Grande Guerre : Les obus miaulent. Cette métaphore courante dans les discours du front et la littérature de guerre s'est figée en cliché. L'écriture de Guillaume Apollinaire, dans sa diversité générique et formelle (correspondance, poésie), met en jeu un processus d'appropriation de l'image, qui manifeste son rôle dans la configuration poétique. Les phénomènes liés à la métaphore illustrent la capacité du discours littéraire à susciter de nouvelles esthésies. Ils interrogent la relation entre figure et visée discursive, entre sens et valeur, à travers l'ethos de l'instance énonciative.

The discursive analysis of rhetorical figures involves a general problematics of the contextualization of linguistic facts, which implies an extensive and interactive definition of the notion of context. The investigation will be based on a Great War image: the mewing of shells. This metaphor, which is frequent in discourse about the front and in war literature, has become a cliché. Guillaume Apollinaire's writing, through its generic and formal diversity (correspondence, poetry), entails an appropriation of this image which plays a part in the poetic configuration. The phenomena linked with the metaphor show the ability that discourse has to create new esthesies. They question the relation between the figure and the discursive aim, between sense and value, through the ethos of the enunciation subject. 
INDEX

Keywords : cliche, configuration, context, contextualization, enunciation, esthesie, ethos, figure, interdiscourse, metaphor

Mots-clés : cliché, configuration, contexte, contextualisation, énonciation, esthésie, ethos, figure, interdiscours, métaphore

\section{AUTEUR}

PHILIPPE WAHL

Passages XX-XXI (EA 4160), Université Lumière Lyon 2 